\title{
Correction
}

\section{Correction: Marginal Likelihood Estimate Comparisons to Obtain Optimal Species Delimitations in Silene sect. Cryptoneurae (Caryophyllaceae)}

\section{The PLOS ONE Staff}

There are several errors in this article. Figure 4 is incorrect. The corrected Figure 4 can be viewed here.
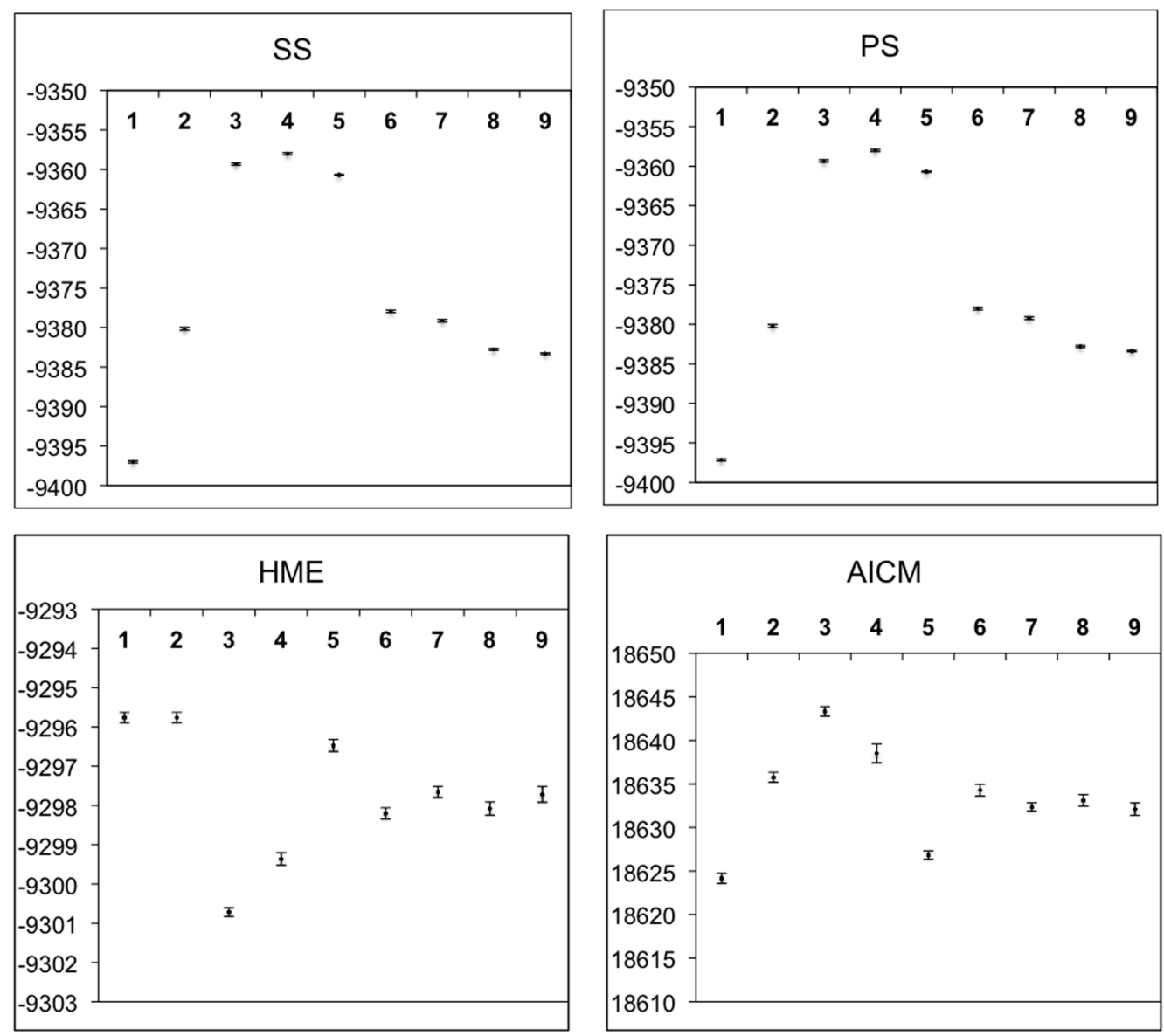

Figure 4. Means and $95 \%$ confidence intervals of marginal likelihood estimates and AICM values estimated from 10 replicate analyses for each of the classification model (1-9). Marginal likelihood were estimated via Path Sampling (PS), Stepping Stone (SS), and Harmonic Mean (HME) methods. AICM (a posterior simulation-based analogue of AIC through MCMC) values were obtained through AICM test. doi:10.1371/journal.pone.0106990.g004

In the second to last paragraph of the Results section, the second sentence is incorrect. The correct sentence is: "HME displayed results that were contradictory to PS and SS, and had much higher means."

In SI Table 1C, the value for the length column of Est 14 is incorrect. The corrected value is 1547 .

\section{Reference}

1. Aydin Z, Marcussen T, Ertekin AS, Oxelman B (2014) Marginal Likelihood Estimate Comparisons to Obtain Optimal Species Delimitations in Silene sect.Cryptoneurae (Caryophyllaceae). PLoS ONE 9(9): e106990. doi:10.1371/journal.pone.0106990
Citation: The PLOS ONE Staff (2014) Correction: Marginal Likelihood Estimate Comparisons to Obtain Optimal Species Delimitations in Silene sect. Cryptoneurae (Caryophyllaceae). PLoS ONE 9(12): e116266. doi:10.1371/journal. pone. 0116266

Published December 17, 2014

Copyright: () 2014 The PLOS ONE Staff. This is an open-access article distributed under the terms of the Creative Commons Attribution License, which permits unrestricted use, distribution, and reproduction in any medium, provided the original author and source are credited. 